\title{
Effects of perceptual quality on hemispheric asymmetries in visible persistence
}

\author{
STEPHEN D. CHRISTMAN \\ University of California, Berkeley, California
}

\begin{abstract}
Two experiments were performed to investigate the effects of input stimuli's perceptual characteristics on visual hemifield asymmetries. The task used involved the temporal integration of form: a stimulus was flashed in two incomplete but complementary flashes, which then had to be integrated over time into the complete stimulus. In this task, successful performance depends on the visible persistence of the first flash. In Experiment 1, an increase in size improved leftvisual-field-right-hemisphere (LVF-RH) performance relative to right-visual-field-left-hemisphere (RVF-LH) performance when the interflash interval (IFI) was 60 msec. When the IFI was increased to $100 \mathrm{msec}$, a change in size had no effect. In Experiment 2, eccentricity of input was manipulated at an IFI of $80 \mathrm{msec}$, and a decrease in eccentricity was found to selectively benefit LVF. $\mathrm{RH}$ performance. These results are interpreted in terms of Sergent's (1983) visual-spatialfrequency-based model of hemispheric lateralization.
\end{abstract}

Recent studies of visual perceptual lateralization have demonstrated that the perceptual quality of a stimulus input can influence observed hemispheric advantages as much as the cognitive nature of the task (e.g., verbal vs. spatial). Studies in which perceptual degradation of stimulus input, independent of stimulus type, resulted in impaired performance in the right visual field-left cerebral hemisphere (RVF-LH) relative to the left visual field-right hemisphere (LVF-RH) were recently reviewed by Sergent (1983b). She specified aspects of perceptual quality closely related to visual spatial frequency components of the stimuli, such as size, retinal eccentricity, masking, and exposure duration. The present experiments were conducted to investigate the effects of perceptual quality on hemispheric superiority of visual persistence, using a task that has been shown to be sensitive to varying spatial frequency components (Di Lollo \& Woods, 1981).

Before discussing the relation of visible persistence to perceptual quality and spatial frequency, it is useful to summarize briefly some information on the effects of stimulus characteristics in the detection of hemifield asymmetries. Sergent, in a series of reviews and experiments (Sergent, 1982b, 1983b, 1984), suggested that the LVF$\mathrm{RH}$ is more efficient at processing lower-spatial-frequency components of visual stimuli and the RVF-LH is more efficient at processing higher-spatial-frequency components (low spatial frequencies correspond to, but are not equivalent to, larger, more global patterns and configu-

\footnotetext{
The author gratefully acknowledges the following individuals: Curtis Hardyck for support and suggestions throughout the project, Steve Lones for the design and construction of the circuit board that drove the LED display, Karen DeValois and Zoe Kersteen for their helpful comments on various aspects of style and methodology, and three anonymous reviewers for useful critiques. This research was supported in part by NIMH Training Grant MH15860-05. Send reprint requests to Stephen Christman, Psychology Department, 3210 Tolman Hall, University of California, Berkeley, CA 94720.
}

rations in a visual scene, whereas high spatial frequencies correspond roughly to smaller, more finely detailed elements of a visual scene). This conception of hemispheric asymmetry conforms to the pattern of results found by investigators who have examined the effects of perceptual quality in the hemifields. Manipulations within language tasks-such as pattern masking, blurring, or increasing the eccentricity of a stimulus (all of which decrease perceptual quality) - are more detrimental to performance in the RVF-LH than to that in the LVF-RH (Hellige, 1984; Hellige \& Webster, 1979; Polich, 1978). If degradation of perceptual quality is defined in the simple sense that the visually identifying features and details of an object cannot be clearly resolved, then it can be argued that it is the relative lack of higher spatial frequencies that leads to greater impairment in the RVF-LH under conditions of degradation.

Many forms of perceptual degradation produce the selective removal of higher-spatial-frequency information from the stimulus. Increasing eccentricity removes high spatial frequencies due to a number of factors, such as imperfections in the optics of the eye and larger receptive fields and sparser distribution of receptors in the parafovea. Similarly, decreasing exposure time prevents the complete resolution of higher frequencies (Breitmeyer \& Ganz, 1977). Different spatial-frequency components of a visual image are extracted and encoded at different rates, with lower spatial frequencies being completely resolved within 50-100 msec, whereas higher spatial frequencies require $100-300 \mathrm{msec}$. If the exposure duration of a stimulus is less than $100 \mathrm{msec}$, the visual information on which cognitive operations must be performed will be carried primarily by the lower spatial frequencies. Therefore, the spatial-frequency hypothesis predicts that LVF-RH performance will be at an advantage at short exposure durations due to the greater sensitivity of the LVF$\mathrm{RH}$ to lower-spatial-frequency information, above and be- 
yond (or below and before) the nature of the task. Conversely, increases in exposure duration will primarily benefit the integration of higher frequencies in the RVF-LH.

The above discussion of the effects of exposure duration assumes that luminance is held constant. The short exposures present in the tachistoscopic paradigm, however, usually fall within the critical duration of Bloch's law. Thus, duration and luminance are reciprocal in determining the extent to which high frequencies are available to the visual system. Just as increasing exposure duration benefits the integration of higher frequencies, so does increasing stimulus luminance. Consequently, the loss of high-frequency information with decreasing exposure duration can be partially counteracted by increasing the luminance.

Because precisely such factors as eccentricity of input, widely varying luminances, and short exposure durations are ubiquitous aspects of tachistoscopic stimulus presentation in laterality work, Sergent (1983b) argued that characteristics of the perceptual input may interact with the cognitive requirements of the task and should always be taken into account when interpreting any results from a tachistoscopic paradigm. For example, Sergent (1982b), using a facial categorization task, found a LVF-RH advantage at a 40-msec exposure duration, no difference at $120 \mathrm{msec}$, and a RVF-LH advantage at an exposure of $200 \mathrm{msec}$. In similar experiments, Bryden and Allard (1976) found that a LVF-RH advantage for certain typefaces with an exposure of $30 \mathrm{msec}$ became insignificant at an exposure of $150 \mathrm{msec}$. In a replication of their experiment, Murray (1982) found a LVF-RH advantage for a specific typeface at a 30 -msec exposure, which became a RVF-LH advantage at $100 \mathrm{msec}$. Regard and Landis (1983), using stenographic characters in a go/no-go task, found a LVF-RH advantage at a 50-msec exposure, no difference at a 100 -msec exposure, and a RVF-LH advantage at a $150-\mathrm{msec}$ exposure.

In a similar manner, retinal eccentricity can interact with the perceptual quality of the input, because the reduced acuity outside the fovea means a corresponding decrease in the amount of available high-spatial-frequency information. Sergent (1983a) varied eccentricity within a lettercomparison task (Posner, Boies, Eichelman, \& Taylor, 1969) and found that increasing distance from fixation impaired performance more for RVF-LH presentations than for LVF-RH ones. Similar results have been obtained using facial stimuli under conditions of increasing eccentricity (Hellige, Corwin, \& Jonsson, 1984, Experiment 3) and low-pass filtering (Jonsson \& Hellige, 1986; Sergent, 1984). Finally, size is also related to the spatial-frequency distributions of stimuli. Doubling the size of a visual image lowers all spatial frequency components by one octave. Sergent (1982a) conducted a visual search experiment using large letters made up of numerous smaller letters. The task was to look for certain target letters, which could be the large ones, the small ones, both, or neither. She found a LVF-RH advantage when the target was one of the large letters, and a RVF-LH advantage when the target was one of the small letters.

Hence, we find equivalent patterns of results that appear to be independent of the analytic or holistic nature of the task. Sergent (1982a) suggested that hemispheric asymmetries are present at levels of processing that are earlier and more sensory-bound than the more abstract levels of cognitive operations focused on by most theories of hemispheric lateralization. She specifically suggested that hemispheric differences initially appear as differential efficiency in processing lower- versus higher-spatialfrequency information.

For the present experiments, the task paradigm of temporal integration was chosen. Temporal integration, a task first developed by Eriksen and Collins (1968), involves the presentation of two successive stimuli, each of which is partial and essentially random, to the same general locus in space. When the two stimuli are combined and integrated over time, a single meaningful stimulus (i.e., a letter, CVC, number, etc.) is obtained from their overlapping percepts. Because the integration cannot occur until the presentation of the second stimulus, the crucial variable in this paradigm is the visible persistence of the first stimulus. For the integration task to be successfully performed, there must be some adequate visual representation of the first stimulus in the system at the time of the second stimulus. The temporal integration task was chosen to investigate the interaction between hemispheric lateralization and perceptual quality/spatial frequency because the duration of visible persistence of stimuli has been found to be strongly related to the range of spatial frequencies, with higher frequencies having a longer persistence than lower frequencies (Di Lollo \& Woods, 1981). Hence, it was expected that performance on this task should be sensitive to manipulations of the first stimulus's spatial frequency components. Furthermore, if there were any difference in hemispheric sensitivity to aspects of the quality and spatial frequency of stimuli, this difference should appear as interactions between quality of input and side of visual hemifield advantage.

Di Lollo (1981) used a temporal integration task to examine hemispheric asymmetries in visible persistence and found none, but there are some possible reasons besides lack of an actual hemispheric asymmetry to account for this result. First, Di Lollo used a very simple sensory task: he required subjects to determine the location of a missing dot in a $4 \times 4$ matrix. Sergent's spatial-frequency hypothesis argues that both hemispheres/hemifields can achieve percepts of equal quality and that "hemispheric differences as a function of spatial frequency must result from processing taking place beyond the sensory level" (Sergent, 1982a, pp. 265-266). A number of recent studies have found no asymmetries between the visual fields in tasks that involved the simple detection of stimuli of varying spatial frequency (Kitterle \& Kaye, 1985; Peterzell, 1984; Previc, 1982). Perhaps Di Lollo's task could be performed purely on the basis of sensory information, without involving any higher order cognitive 
processes, such as discrimination or memory. A second problem with Di Lollo's findings is the fact that absolute effects are hard to interpret. There is undoubtedly more occurring in the process of visible persistence than the simple analysis of high versus low spatial frequencies, and of course there is more to the bases of cerebral lateralization than the spatial-frequency hypothesis alone. It could be the case that in Di Lollo's experiment, visible persistence was longer and/or of higher resolution in the RVF$\mathrm{LH}$, but some other factor about the integrative nature of the task or short duration of the stimulus might have been biased toward better performance in the LVF-RH, in which case the two would cancel each other out and any effect would be obscured.

The present study tried to avoid this problem by looking for a relative effect. Digits were used in the integration paradigm in an effort to tap mental processes beyond the sensory level. Specifically, a visual integration task was performed on blocks of higher-spatial-frequency trials versus lower-frequency trials. No a priori prediction about the absolute visual field advantage was made; the crucial test would be the presence of a visual field $\times$ spatial frequency interaction. A specific prediction was that as the relative proportion of higher-spatial-frequency components of stimuli were decreased (by increasing the size or eccentricity), there should be an accompanying shift toward better performance in the LVF-RH.

\title{
EXPERIMENT 1
}

\begin{abstract}
Method
Apparatus. The subjects sat in a darkened room and viewed the display through a viewing hood that was attached to a chair, ensuring that the subject's midline was correctly aligned with the center of the display. The viewing hood also served to minimize head movement, while still allowing the subject to speak. To ensure parafoveal presentation of the stimuli, eye fixation was monitored by an Applied Sciences eye-movement monitoring system. At the beginning of a test session, the subject fixated on a point in the center of the LED display, and the digital $x$ and $y$ coordinates of fixation were determined. At the start of each test trial, the coordinates of the subject's fixation were taken and compared to values obtained during the initial alignment. Eye position was sampled every $16.7 \mathrm{msec}$. The trial was not initiated until the eye-tracker reported five successive samplings to be within $0.25^{\circ}$ of visual angle of the central fixation coordinates. Multiple sampling ensured that stimuli would not be presented in the course of a saccade across the fixation point.

Visual Display. The display consisted of two standard sevenelement LEDs, one in the LVF and the other in the RVF. Spatial frequency of the stimuli was manipulated by changing display size: the lower frequency stimuli were twice the size of the higher frequency stimuli. The LEDs were $1.9 \mathrm{~cm}$ high and $1.25 \mathrm{~cm}$ wide, subtending $1.2^{\circ} \times 0.8^{\circ}$ of visual angle in the high-spatial-frequency condition and $2.4^{\circ} \times 1.6^{\circ}$ of visual angle in the low-frequency condition. The size of the displays was manipulated by varying the distance between the display and the subject. The display was $46 \mathrm{~cm}$ away from the subject in the low-frequency (large size) condition, and $91 \mathrm{~cm}$ away in the high-frequency (small size) condition. In both conditions the display was located $3^{\circ}$ parafoveally from the fixation point, which was a nonluminous orange spot. Display luminance was $1.5 \mathrm{fL}$, with a background illumination of $0.2 \mathrm{fL}$. Dis-
\end{abstract}

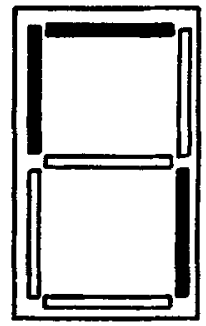

(A)
(B)

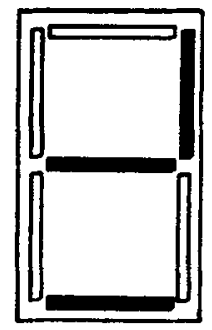

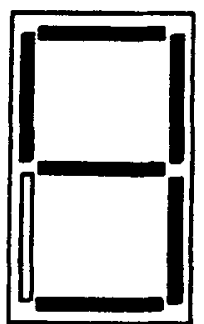

(C)
Figure 1. LED stimulus display: (A) first flash; (B) second flash; (C) integrated percept of the number 9 .

plays were generated by a PDP-11 computer, which also performed all scoring and timing functions.

Design and Procedure. At the beginning of each trial, a semirandom subset of one to four of the seven elements in either the LVF or the RVF would light up for $4 \mathrm{msec}$, followed by a blank interflash interval (IFI) of $60 \mathrm{msec}$ in one condition and $100 \mathrm{msec}$ in the other, after which a second flash was presented on the same side for another $4 \mathrm{msec}$ (these temporal parameters were chosen to avoid floor and ceiling effects). The two flashes never shared any common elements and were complementary in that when overlaid and integrated, a single digit between zero and nine was formed (Figure 1). The randomness of the flashes was constrained so as to avoid the occurrence within a single flash of possibly biasing or informative cues (e.g., contiguous corners or connected lines). The number of elements lit in each flash was held roughly constant; that is, the number of lit elements in the second flash equaled the number of lit elements in the first flash plus or minus one. The subject's task was to recognize and report the number. A headphonemicrophone set allowed for communication between the subject and the experimenter, who sat at a computer console and entered the subject's responses on a keyboard.

Three independent variables were manipulated: visual field of presentation, relative balance of high- versus low-frequency components of the stimuli (this was done by varying stimulus size), and IFI. IFI length was a between-subjects variable, and the other two were within-subject variables. IFI was chosen as an experimental variable to ensure that this task was a measure of visible, as opposed to schematic, persistence. Visible persistence can be seen and is short-lived, lasting approximately $100-200 \mathrm{msec}$, whereas schematic persistence is nonvisible, consists of lower quality structural and schematic information, and lasts for up to $600 \mathrm{msec}$ (Phillips, 1974). Under conditions of visible persistence, there should be a large drop in performance at an IFI of $100 \mathrm{msec}$. The desire to establish the visible nature of the persistence in this task was motivated by a current controversy as to whether the laterality of visual aftereffects occurs with visible as well as schematic persistence (Di Lollo, 1983; Wurst \& Long, 1983).

Each subject participated in one of the IFI conditions for a total of 60 trials in each of four conditions yielded by the factorial combination of two levels of visual field and two levels of spatial frequency distribution. Test trials were presented in two blocks of 120 trials each; in each block, the size of the stimulus was held constant while trials varied randomly between the LVF and the RVF ( 60 per side). Each subject was also given 20 practice trials prior to the test trials to familiarize him/her with the nature of the stimuli.

In addition, two control conditions were run. First, a condition was run in which the whole digit was presented (at the same size as in the low-frequency condition) in a single flash lasting $2 \mathrm{msec}$. This duration was chosen, after the collection of pilot data, to provide a level of performance comparable to that in the test conditions. This condition was added to test for any inherent visual-field 
advantage for the numeric stimuli. The order of presentation of the high-frequency, low-frequency, and control conditions was counterbalanced across subjects. The total session lasted for about $50 \mathrm{~min}$.

A second control condition was run to assess the approximate level of baseline performance on the task and to determine whether there was any hemispheric difference in the ability to identify the stimuli on the basis of partial information (cf. Nebes, 1971). To address these issues, a condition was run in which only the second of the two flash stimuli from the test conditions was presented to the LVF and RVF for $4 \mathrm{msec}$, and the subject's guesses at which digit each flash could be a subset of were compared with the correct answers (given both flashes) from the test condition to arrive at a percentage correct score. Due to a rearrangement of the subject room, the display was farther away from the subject for this condition only, and the digits subtended $.8^{\circ} \times .53^{\circ}$ of visual angle.

Subjects. Forty subjects, 20 female and 20 male, participated in the test conditions and whole-digit control condition of this experiment as part of the requirements for an undergraduate introductory psychology course. An additional 10 subjects participated in the partial-flash condition. All subjects were right-handed and had no left-handed relatives in their immediate families. All subjects were also naive psychophysical observers.

\section{Results}

The measure of performance for each subject was the percentage of correct responses for each visual field per condition. These scores, averaged over the 20 subjects for each IFI, are shown in Figure 2. A repeated measures analysis of variance was carried out. The between-subjects comparison of the two different IFI conditions yielded a strong effect of IFI $[F(1,38)=77.2, p<.0001]$, with performance better at the 60 -msec IFI. There was also an overall effect of visual field $[F(1,38)=6.90$, $p<.02$ ], with the the LVF-RH superior. An analysis of simple main effects showed that this LVF-RH advantage was significant only in the 100 -msec IFI condition $[F(1,18)=6.92, p<.02]$. At a 60 -msec IFI, there was no simple main effect of visual field $[F(1,18)=1.52$, $p>.23]$. There was no overall effect of size $[F(1,38)$ $<1]$. There were no significant effects of gender $(F<1)$, so all further comparisons were collapsed across sex. The only other significant effect for the combined IFI comparison was a three-way interaction among visual field, stimulus size, and length of IFI $[F(1,38)=5.03$, $p<.05]$. An analysis of simple effects showed that this interaction arose from a significant interaction between visual field and size at an IFI of $60 \mathrm{msec}[F(1,18)=6.12$, $p<.025]$, but not at an IFI of $100 \mathrm{msec}[F(1,18)<1]$. Specifically, as the stimulus size was increased in the 60 msec IFI condition, performance was impaired more in the RVF-LH than in the LVF-RH.

Apart from this interaction between size and visual field, there were no significant effects of size, although at the 60-msec IFI there was a weak trend toward better performance with smaller stimuli $[F(1,18)=2.60, p<.13]$. This result may seem slightly counterintuitive, since one might expect better performance with larger stimuli. Insofar as reducing the size of stimuli increases the proportion of high, relative to low, frequencies, however, this

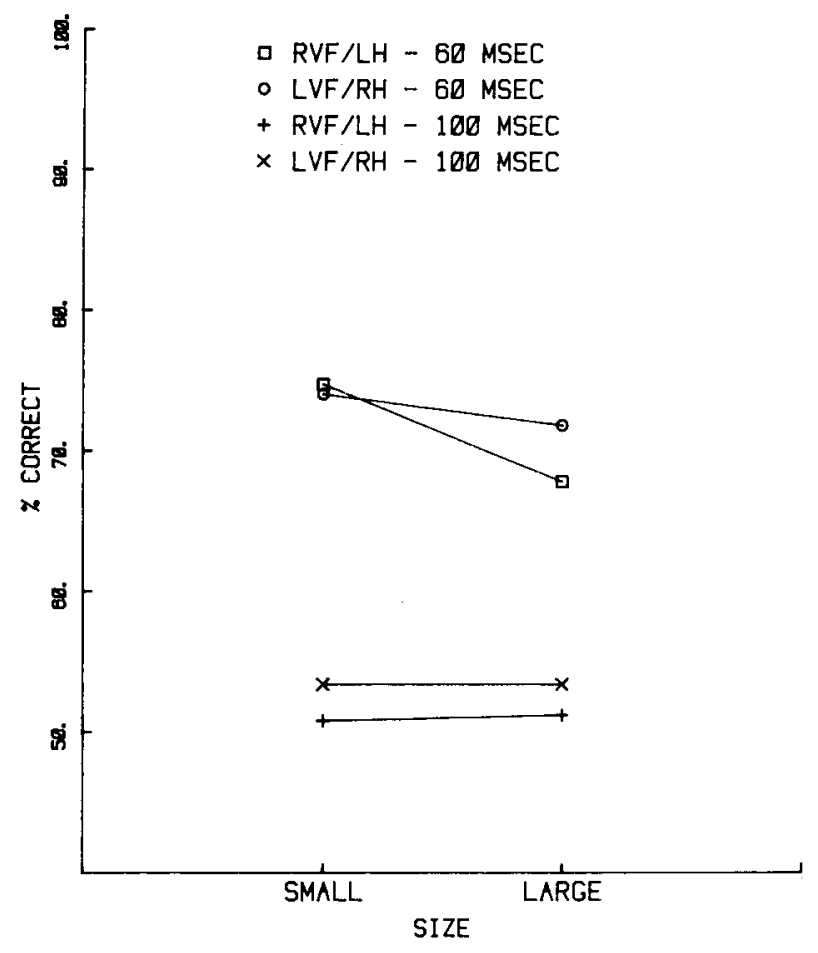

Figure 2. Percentage correct performance as a function of interstimulus interval, size of stimulus, and visual field of presentation.

result supports findings of longer visible persistence at higher frequencies (Di Lollo \& Woods, 1981).

Finally, the whole-digit control condition showed no differences between the two sides $[F(1,39)<1]$. The level of accuracy was $79.7 \%$ in the LVF-RH and $80.3 \%$ in the RVF-LH. This result is useful in that it demonstrates that the accuracy for the baseline process of recognizing briefly flashed numbers on LEDs is equal in the two hemispheres. Similarly, there was no significant difference between the visual fields in the partial-flash condition, although there was a weak trend toward a RVF-LH advantage $[F(1,9)=2.30, p<.18]$. This suggests that there is no hemispheric asymmetry in the ability to reconstruct the stimuli from partial information.

\section{Discussion}

The expected interaction between visual field and size (as related to spatial frequency components) was significant in the 60-msec IFI condition. As the proportion of high, relative to low, spatial frequencies was increased, performance in the RVF-LH improved, whereas performance in the LVF-RH was unaffected. Hence, it appears that the RVF-LH was able to make more efficient use of the increased distribution of high-spatial-frequency components in the small-size condition. This is in accordance with Sergent's spatial-frequency hypothesis (Sergent, 1982b). It should be mentioned that there was an absolute trend toward a LVF-RH advantage, especially in the large size (low-frequency) condition. Because accurate 
performance on the visible persistence task is ostensibly loaded on the higher frequencies, a RVF-LH advantage might be expected. Absolute effects are hard to interpret, however, because of the great number of procedural and stimuli-related factors that may affect and confound results in divided hemifield experiments. For example, the stimuli in the present study were presented for relatively short durations and at low luminance, both of which tend to result in LVF-RH advantages in similar tasks (Sergent, 1983b). The important point is that the two hemispheres were differentially benefited by changes in the spatial frequency components of the stimuli, independent of the nature of the task.

In the 100-msec IFI condition, there were no effects of gender or stimulus size $[F(1,18)<1]$, and no interactions reached significance. The only significant effect was of visual field of presentation $[F(1,18)=6.92$, $p<.02]$, with performance slightly and consistently better in the LVF-RH. Lack of significance in this condition may have been due to floor effects. Accuracy was much lower than in the 60-msec IFI condition, and many subjects commented on the task's difficulty, often showing surprise that they were getting any correct. Unfortunately, it is virtually impossible to precisely compute what an overall level of chance performance would be on this task, because some numbers that are simple patterns on an LED (e.g., 1 and 7) were almost always correctly responded to, whereas other, more visuospatially complex, digits (e.g., 6, 8, 9) were much more difficult. Table 1 shows the accuracy level for individual digits in both test conditions of Experiment 1 and in Experiment 2. It can be seen that accuracy levels in the 100 -msec condition, for example, ranged from perfect performance to true chance performance $(10 \%)$. In addition, the second flash, even in the absence of any information from the first flash, could be used to constrain the possible guesses. Hence, chance performance could be better than simply 1 in 10 . The partial-flash condition yielded an overall level of performance of around $30 \%$ correct. Although this is below the $50 \%$ level found in the $100-\mathrm{msec}$ condition, it is close enough to support the conclusion that the current temporal integration task tapped into processes of visible, not schematic, persistence. Visible persistence has a duration of 100-200 msec (Turvey, 1978), and it seems reason-

Table 1

Task Performance in Experiments 1 and 2 Broken Down for Individual Digits in Terms of Percentage Correct

\begin{tabular}{|c|c|c|c|c|c|c|c|c|c|c|}
\hline \multirow[b]{2}{*}{ Condition } & \multicolumn{10}{|c|}{ Individual Digit } \\
\hline & 1 & 2 & 3 & 4 & 5 & 6 & 7 & 8 & 9 & 0 \\
\hline \multicolumn{11}{|l|}{$60-\mathrm{msec}$ IFI } \\
\hline Large & 98 & 89 & 63 & 96 & 85 & 54 & 96 & 16 & 34 & 63 \\
\hline Small & 98 & 91 & 74 & 94 & 80 & 62 & 97 & 29 & 47 & 72 \\
\hline \multicolumn{11}{|l|}{ 100-msec IFI } \\
\hline Large & 100 & 71 & 30 & 75 & 66 & 26 & 95 & 15 & 14 & 31 \\
\hline Small & 98 & 61 & 45 & 71 & 58 & 27 & 90 & 10 & 20 & 39 \\
\hline \multicolumn{11}{|l|}{ 80-msec IFI } \\
\hline Outer & 98 & 76 & 53 & 87 & 74 & 32 & 94 & 12 & 20 & 51 \\
\hline Inner & 99 & 87 & 48 & 88 & 76 & 37 & 98 & 15 & 26 & 43 \\
\hline
\end{tabular}

able to conclude that as the length of the IFI approaches $200 \mathrm{msec}$, performance will approach the $30 \%$ level.

An issue that is raised, although not resolved, by these findings is whether the current task measures Type I or Type II persistence, as defined by Long (1985). Long classified the successive-field type of integration task as an example of Type II persistence, which is characterized by, among other things, positive size effects (larger stimuli having longer persistence). The present experiment, however, found an inverse size effect, which is defined by Long as being a hallmark of Type I persistence. A possible reason for this inconsistency may be that the experiments using the successive-field integration tasks that Long classified as being examples of Type $\Pi$ persistence were characterized by a large set of alternative responses: Eriksen and Collins (1968) had their subjects identify a CVC nonsense syllable, and Kinnucan and Friden (1981) had their subjects identify the missing dot in a $5 \times 5$ dot matrix. The present study, on the other hand, had a much smaller set of possible responses; in addition, the use of the LED display creates digits that are somewhat artificial in appearance and are potentially more confusable with one another. Hence, the stimuli in the present experiment may have required a greater degree of integration in order to enable the subject to discriminate the correct response from the limited set of similar alternatives. Thus, it may be that Type I persistence represents the situation in which the integration of two successive flashes is relatively complete, whereas Type II persistence represents the situation in which the discontinuity between the flashes is apparent, but the persistence is still of high enough quality for successful integration under certain circumstances.

\section{EXPERIMENT 2}

The purpose of Experiment 2 was to determine whether changes in the eccentricity of the stimulus input would have effects on performance of the integration task in the two visual fields similar to the effects of size changes in Experiment 1 . Of the many aspects of tachistoscopic input that are closely related to spatial-frequency distributions, perhaps none is as "hidden" and taken for granted as eccentricity. Of course, divided-visual-field work requires lateralization, and hence eccentricity, of input. Unfortunately, this has often meant that researchers have not felt the need to consider any possible effects that arise specifically from the eccentricity of the input, above and beyond any effects arising from higher-level factors. Experiment 2 sought to determine the nature of the effects of varying eccentricity on performance, with other aspects of the stimuli and task remaining constant.

\section{Method}

Experiment 2 differed from Experiment 1 in two ways. First, the effective spatial-frequency components of the stimuli were manipulated by changing retinal eccentricity of the input instead of size. Display dimensions were the same as in Experiment 1 . The highspatial-frequency condition for this experiment was equivalent to 
the low-frequency (large size) condition in Experiment 1: the display was $46 \mathrm{~cm}$ away from the subject and was located $3^{\circ}$ parafoveally from the fixation point. For the low-frequency condition, the display was moved out to $6^{\circ}$ eccentricity while remaining $46 \mathrm{~cm}$ away from the subject. Increasing eccentricity decreases the relative proportion of high spatial frequencies available to the visual system, due to the less dense receptive-field distribution (leading to poorer resolution) in the periphery of the visual field. The second difference in this experiment was the use of an 80-msec IFI. This value was chosen to avoid the floor effects in the 100 -msec IFI condition of Experiment 1.

\section{Results and Discussion}

The results of Experiment 2 are shown in Figure 3. The expected interaction between visual field and eccentricity (spatial frequency) was significant $[F(1,18)=6.08$, $p<.03]$. The direction of the interaction, however, was opposite to that found in Experiment 1. When the eccentricity of the input was increased, thus decreasing the proportion of high spatial frequencies, performance dropped sharply in the LVF-RH while staying constant in the RVF-LH. An analysis of simple effects showed a significant LVF-RH advantage at $3^{\circ}$ eccentricity $[F(1,18)$ $=5.60, p<.05]$, with no difference between the visual fields at $6^{\circ}$ eccentricity $[F(1,18)<1]$. When size was increased in Experiment 1, also resulting in a decreased proportion of high spatial frequencies, performance was more impaired in the RVF-LH. There were no main effects of visual field or eccentricity.

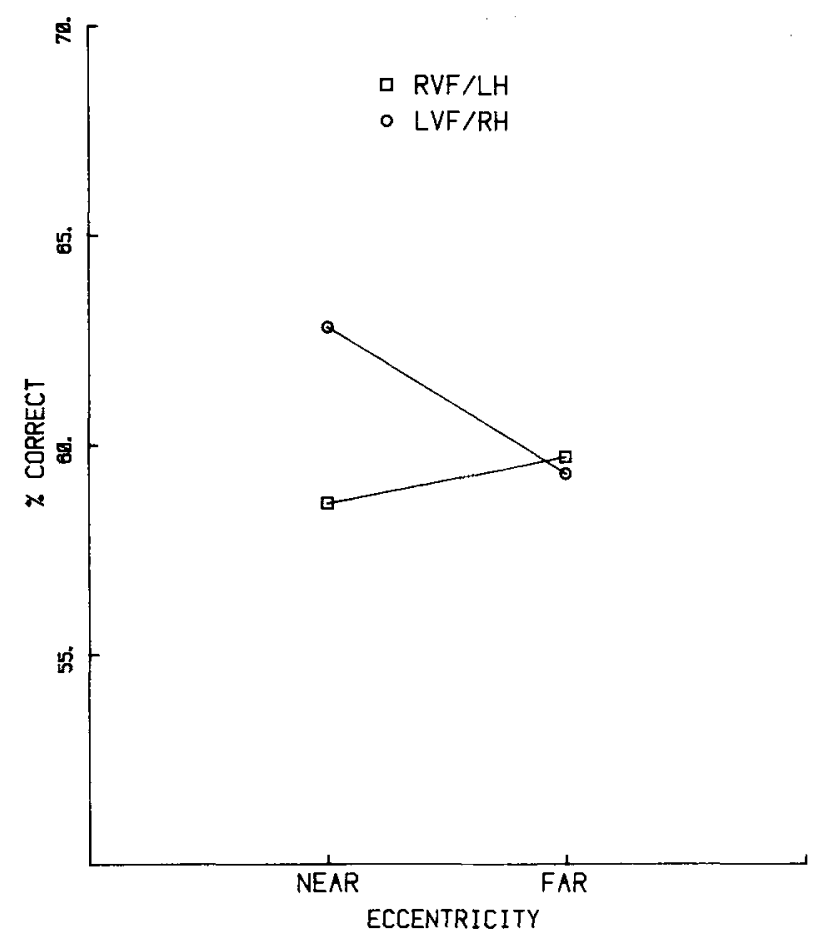

Figure 3. Percentage correct performance as a function of eccentricity and visual field of presentation.

\section{GENERAL DISCUSSION}

The intent of the present experiments was to investigate the effects of input characteristics on hemispheric lateralization, especially insofar as those characteristics interact with spatial-frequency components. Experiment 1 examined the effects of the size of the input and found that when stimulus size was doubled (thus lowering the spatialfrequency components by one octave), performance was more impaired in the RVF-LH than in the LVF-RH. This is in accordance with the spatial-frequency hypothesis, inasmuch as the LVF-RH was better able to take advantage of increased low frequencies (and conversely was less impaired by a decrease in high frequencies) than was the RVF-LH. It should be noted that this effect was obtained with a relatively small change in size (doubling). In Sergent's (1982b) report of a LVF-RH advantage for large letters and a RVF-LH advantage for small ones, the large letters were approximately 10 times larger than the small ones. The results for the 100-msec condition of Experiment 1 proved inconclusive. There may have been a floor effect in performance, which could have prevented the appearance of any interaction between visual field and size of input. The control conditions of Experiment 1 supported the notion that performance in the $100-\mathrm{msec}$ condition indeed approached a lower asymptotic level of performance, suggesting that the task involved visible persistence.

Experiment 2 investigated the effects of eccentricity on the visible persistence task. Typically, increases in eccentricity result in greater impairment in the RVF-LH than in the LVF-RH. Sergent (1983a) found greater impairment of RVF-LH performance with increasing eccentricity in a letter-comparison task. Polich (1978) found an equivalent pattern of results with a less verbal task. The present results, unexpectedly, were in the opposite direction, with increasing eccentricity resulting in greater impairment in the LVF-RH. This outcome apparently contradicts the spatial-frequency hypothesis, since it might be expected that decreases in available high frequencies with increasing eccentricity should impair the RVF-LH more than the LVF-RH.

In a recent study, Hellige et al. (1984) found a related pattern of results that helps reconcile the current findings with the spatial-frequency hypothesis. Hellige et al. found that both increasing eccentricity and increasing perceptual degradation (resulting in a decrease in available higher spatial frequencies) resulted in greater relative impairment of LVF-RH performance in a task that required the discrimination of a set of male target faces from a set of male distractor faces. This result was unexpected. In the same study, however, another condition resulted in the expected pattern of greater RVF-LH impairment with increasing stimulus degradation for a task involving the discrimination of male target faces from a set of female distractor faces. The clue to explaining these seemingly contradic- 
tory results lies in the fact that there is another factor operating beyond the quantitative nature of the input and the qualitative nature of the task: the interaction between the level of feature analysis required by the task and that allowed by the input. The male/female discrimination task might have been more easily performed on the basis of global, low-spatial-frequency aspects of the stimuli, such as shape of hair or outline of jaw. When all the faces were male, however, there was less perceptual distinguishability between the targets and the distractors, and the discrimination may have required the processing of more detailed, high-spatial-frequency features, such as distance between eyes or shape of nose. Successful performance on the visible persistence task is similarly loaded on higher frequencies, in that lower frequencies may have insufficient persistence to contribute to task performance.

Thus, in both Hellige et al.'s (1984) male/male discriminations and the present Experiment 2, the information carried by the remaining high frequencies may have been at a premium, and the LVF-RH may have suffered in performance due to its relative lack of sensitivity to high-spatial-frequency components, whereas the RVF-LH was able to extract enough high-frequency information to outperform the LVF-RH. Hellige et al. arrived at a similar conclusion: insofar as "the various attributes of a task converge to make the processing of local features a necessity, the right hemisphere is at something of a disadvantage when those local features become difficult to extract" (Hellige et al., 1984, pp. 105-106). Sergent (1985) found a similar task $\times$ visual field interaction, and stressed the importance of the way in which the spatial frequencies available in the stimulus will interact with the spatial frequencies required for processing in determining relative hemispheric superiority.

The question remains, however, of why a change in eccentricity in Experiment 2 should result in an interaction in the opposite direction to the one caused by the change in size in Experiment 1. An answer is suggested by the different ways in which the relative proportions of high and low frequencies are affected by changes in size versus changes in eccentricity. When size is increased, all of the frequency components are shifted down in the frequency domain, but the relative shape of the distribution remains the same. Increases in eccentricity, on the other hand, tend to selectively remove higher frequencies while leaving lower frequencies intact. Hence, increasing eccentricity changes the shape of the frequency distribution, shifting toward an increased proportion of lower frequencies, whereas increasing size preserves the proportion of low to high frequencies, but with a lower overall mean for the distribution.

Thus, when size was increased in the first condition of Experiment 1, the resulting shift of all components toward a lower mean frequency may have allowed superior LVFRH performance of the task on the basis of intermediate to lower frequencies (with little decrement caused by the decrease in high frequencies). In Experiment 2, however, the increase in eccentricity resulted in the selective removal of the intermediate and higher frequencies necessary for the successful performance of the persistence task, leaving intact the lower frequencies that were not as helpful for task performance, especially at the longer IFI of $80 \mathrm{msec}$. Under these conditions, a premium is placed on whatever remaining high-frequency information is left available in the stimulus, and increased relative sensitivity to high frequency components may have enabled RVFLH performance to be less impaired by the increase in eccentricity.

One last aspect of the results that should be discussed is their relation to previous investigations of laterality effects in visible persistence in tasks that did not involve temporal integration. Cohen (1976) and Marzi, Di Stefano, Tassinari, and Crea (1979) used partial-report tasks (see Sperling, 1960). Cohen found longer persistence in the LVF-RH. Long (1985) classified partial-report tasks as measuring Type II persistence, for which lower spatial frequencies have a longer persistence. Although the discussion of Experiment 1 raises some question about the Type I/Type II distinction, Cohen's finding of longer persistence in the RH suggests that her task did indeed measure Type II persistence (whereas the present task measures Type I). Marzi et al. also used a partial-report task, but failed to find any hemispheric asymmetry. Schmuller and Goodman (1980) studied persistence in the two visual fields by presenting two outline drawings of simple objects, one in each visual field, followed by an arrow cuing order of report. They found a smaller difference between first and second report scores for the RVFLH, suggesting faster decay (i.e., shorter persistence) in the LVF-RH. This finding is in accord with the present results, although Schmuller and Goodman's method of estimating persistence was indirect, and their results could be attributed to more efficient object recognition abilities in the RVF-LH (especially with the use of line drawings, which have a higher proportion of high-spatial-frequency components than do photographs). Long (1984) employed a judgment of synchrony task and found no evidence of hemispheric asymmetry. This lack of asymmetry may be attributable to the fact that the task was very simple and required little more than detection of stimulus offset.

The results of the present study provide evidence of hemispheric asymmetry in a visible persistence task and offer moderate and conditional support for Sergent's (1982a, 1983b) spatial-frequency hypothesis. Visual field $X$ spatial frequency interactions were obtained in two different conditions. The unexpected direction of the interaction when eccentricity was manipulated, however, shows that the picture is more complex than a simple model postulating that the right hemisphere is better with low frequencies, the left with higher frequencies. Aspects of the quality of the stimulus interact with task requirements, producing effects at the level of feature analysis required for successful task performance. For instance, there is no unitary mechanism or process in the hemispheres for recognizing faces. A face can be recognized on the basis of local or global features. Aspects of 
the task (e.g., difficulty of required discrimination) and/or of the stimulus (e.g., degradation) will interact to determine the nature of the visual field $\times$ spatial frequency interaction.

The spatial-frequency hypothesis has two very important implications for laterality researchers. First, the specific physical parameters used in divided-visual-field work, which researchers have tended to ignore, need to be explicitly taken into account when interpreting findings. Second, the idea of asymmetries based on spatialfrequency processing in the visual domain could be extrapolated to other sensory domains (e.g., auditory, tactile, motor) in terms of spatiotemporal frequency processing.

\section{REFERENCES}

Breitmeyer, B., \& GANZ, L. (1977). Temporal studies with flashed gratings: Inferences about human transient and sustained channels. Vision Research, 16, 861-865.

BRYDEN, M., \& ALLARD, F. (1976). Visual hemifield differences depend on typeface. Brain \& Language, 3, 191-200.

CoHen, G. (1976). Components of the laterality effect in letter recognition: Asymmetries in iconic storage. Quarterly Joumal of Experimental Psychology, 28, 105-114.

DI LoLLo, V. (1981). Hemispheric symmetry in duration of visible persistence. Perception \& Psychophysics, 29, 21-25.

Di Lollo, V. (1983). On laterality of visual aftereffects: A rejoinder. Perception \& Psychophysics, 33, 599-603.

Di LoLLo, V., \& WoODs, E. (1981). Duration of visible persistence in relation to range of spatial frequencies. Journal of Experimental Psychology: Human Perception \& Performance, 7, 754-769.

ERIKSEN, C., \& Collins, J. (1968). Sensory traces versus the psychological moment in the temporal organization of form. Journal of Experimental Psychology, 77, 376-382.

Hellige, J. (1984). Perceptual quality and cerebral laterality: Theoretical implications. Paper presented at the Symposium on Cognitive Models of Cerebral Lateralization at the Seventh European Conference of the International Neuropsychological Society, Aachen.

Hellige, J., Corwin, W., Jonsson, J. (1984). Effects of perceptual quality on the processing of human faces presented to the left and right cerebral hemispheres. Journal of Experimental Psychology: Human Perception \& Performance, 10, 90-107.

Hellige, J., \& Webster, R. (1979). Right hemisphere superiority for initial stages of letter processing. Neuropsychologia, 17, 653-660.

Jonsson, J., \& Hellige, J. (1986). Lateralized effects of blurring: A test of the visual spatial frequency model of cerebral hemisphere asymmetry. Neuropsychologia, 24, 351-362.

KinnuCAN, M., \& FrIDEN, T. (1981). Visual form integration and discontinuity detection. Journal of Experimental Psychology: Human Perception \& Performance, 7, 948-953.

KitTerle, F., KAYE, R. (1985). Hemispheric symmetry in contrast and orientation sensitivity. Perception \& Psychophysics, 37, 391-396.

LoNG, G. (1984). Laterality and visual persistence: Support for Di Lollo's model of hemispheric symmetry. Bulletin of the Psychonomic Society, 22, 443-445.
LoNG, G. (1985). The varieties of visual persistence: Comments on Yeomans and Irwin. Perception \& Psychophysics, 38, 381-385.

Marzi, C., Di Stefano, M., Tassinari, G., \& Crea, F. (1979). Iconic storage in the two hemispheres. Joumal of Experimental Psychology: Human Perception \& Performance, 5, 31-41.

MURRAY, J. (1982). The effects of exposure duration on visual field differences in letter identification. Unpublished master's thesis, University of Waterloo, Canada.

Nebes, R. (1971). Superiority of the minor hemisphere in commisurotomized man for the perception of part-whole relations. Cortex, 13, 333-349.

PeTERZELL, D. (1984). Spatial frequencies and the cerebral hemispheres: Identification of digitally filtered letters. Unpublished manuscript, University of Colorado, Boulder.

PHiLlips, W. (1974). On the distinction between sensory storage and short-term visual memory. Perception \& Psychophysics, 16, 282-290.

PoLICH, J. (1978). Hemispheric differences in stimulus identification. Perception \& Psychophysics, 24, 49-57.

Posner, M., Boies, S., Elchelman, W., \& TAylor, R. (1969). Retention of visual and name codes of single letters. Journal of Experimental Psychology, 79, 1-13.

Previc, F. (1982). Visual pattern recognition in the cerebral hemispheres: The role of spatial filtering. Perceptual \& Motor Skills, 55, 1319-1326.

Regard, M., \& LANDIs, T. (1983). Dissociated hemispheric superiorities for reading stenography versus print. Paper presented at the International Neuropsychology Society Sixth European Conference, Lisbon.

Schmuller, J., \& Goodman, R. (1980). Bilateral tachistoscopic perception, handedness, and laterality: II. Nonverbal stimuli. Brain \& Language, 11, 12-18.

SERGENT, J. (1982a). The cerebral balance of power: Confrontation or cooperation? Journal of Experimental Psychology: Human Perception \& Performance, 8, 253-272.

SERGENT, J. (1982b). Theoretical and methodological consequences of variations in exposure duration in laterality studies. Perception \& Psychophysics, 31, 451-461.

SERGENT, J. (1983a). The effects of sensory limitations on hemispheric asymmetries. Canadian Journal of Psychology, 37, 345-366.

SERGENT, J. (1983b). Role of input in visual hemispheric asymmetries. Psychological Bulletin, 93, 481-512.

SERGENT, J. (1984). Sensory limitations and hemispheric asymmetries in the processing of faces. Paper presented at the Symposium on Cog nitive Models of Cerebral Lateralization at the Seventh European Conference of the International Neuropsychological Society, Aachen.

SERGENT, J. (1985). Influence of task and input factors on hemispheric involvement in face processing. Journal of Experimental Psychology: Human Perception \& Performance, 11, 846-861.

SPERLING, G. (1960). The information available in brief visual presentation. Psychological Monographs, 74, 498.

TURVEY, M. (1978). Visual processing and short-term memory. In W. K. Estes (Ed.), Handbook of learning and cognitive processes (Vol. 5). Hillsdale, NJ: Erlbaum.

WURST, S., \& LONG, G. (1983). Laterality and visual persistence: Still a two-sided issue. Perception \& Psychophysics, 33, 595-598.

(Manuscript received December 24, 1985; revision accepted for publication December 22, 1986.) 